\title{
Cognitive Radio Spectrum Sensing Technique Using M-ary QAM for Different Fading Environments
}

\author{
Jai Sukh Paul Singh ${ }^{1}$, Mritunjay Kumar Rai ${ }^{1}$, Gulshan Kumar ${ }^{2 *}$ and Hye-jin Kim ${ }^{3}$ \\ ${ }^{1}$ School of Electronics and Communication Engineering, Lovely Professional \\ University, \\ ${ }^{2}$ School of Computer Science Engineering, Lovely Professional University, \\ ${ }^{3}$ Business Administration Research Institute, Sungshin W. University, Seoul, \\ Republic of Korea \\ khalsa128@gmail.com,raimritunjay@gmail.com,gulshan3971@gmail.com, \\ Hyejina@daum.net
}

\begin{abstract}
Cognitive Radio have emerged up as the innovative technology to counter the problem of Spectrum Scarcity and it's under utilization. Using Cognitive Radio unused spectrum bands by their licensed users can be utilized by the secondary users and therefore increase the overall spectrum utilization. In this paper energy detection based Spectrum Sensing Technique is proposed based on M-ary QAM. The proposed techniques works under diverse channel conditions and environments. To test and verify the proposed technique, Receiver Operating Characteristics (ROC) curves for analysing Probability of Detection $(P d)$ and Complimentary ROC curves for Probability of Missed Detection (Pmd) is performed. The proposed technique is tested over different fading channels with varying Signal to Noise Ratio.
\end{abstract}

Keywords: Cognitive Radio, Primary User, Spectrum, M-ary QAM

\section{Introduction}

Day by day increase in demand high speed data for wireless devices, the necessity of broad spectrum bands is required. The availability of spectrum bands cannot be increased. So to provide solution to such a problem various techniques have proposed so that over the prefixed spectrum band more data rates can be achieved. Moreover, by the FCC report 2005 , only $25 \%$ of the spectrum allocated to the licensed user is utilized, thereby leading to the problem of spectrum under-utilization. So this can be said that shortage of spectrum is not due to fundamental lack of spectrum but due to ineffective use to spectrum. In short, the radio spectrum suffers due to improper utilization by the static allocation of bands to the Licensed User or so called Primary Users (PU). To overcome this issue of spectrum scarcity and it's under-utilization, Cognitive Radio (CR) has emerged as an innovative futuristic technology [1]. CR allows the non-licensed users also known as Secondary Users (SU) or Cognitive Users (CU) to opportunistically access the licensed spectrum bands without interfering with the Primary User services. In this case the Quality of Service (QoS) of both PU as well as the Secondary User is maintained.

Cognitive Radio works four basics blocks. Firstly, Spectrum Sensing, Secondly, Spectrum Management, Thirdly, Spectrum Sharing and Fourthly Spectrum Mobility. In Spectrum Sensing, idle spectrum spaces are detected. This free spectrum spaces are known as spectrum holes or White Spaces. After the sensing management of spectrum is done in which according to the demand of the user best spectrum is selected. After the selection of particular spectrum band, sharing of data over the allocated spectrum band take place by the secondary users. This is transferring of data is known as Spectrum 
Sharing. Sharing continues until the whole data to be transferred in completed or until the emergence of Primary User. With the immediate emergence of Primary User, Secondary User have to vacate the accessed spectrum band immediately and shift over the other free spectrum band. This shifting within the vacant spectrum band is known as Spectrum Mobility.

There are three types of approaches over which Cognitive Radio works i.e. Overlay Approach, Underlay Approach and Interweave Approach. In the overlay approach Secondary User act as a helping hand by providing assistant to the Primary Users to complete their task quickly. After their completion Secondary User is allowed to access the licensed spectrum bands. Whereas in Underlay approach both the Primary User and Secondary User can use the spectrum simultaneously using different transmitting powers. In this case generally secondary user transmit at low power thereby maintaining the level of interference below the predefined threshold. Both the overlay and underlay approach have significant more disadvantages like more delay, interference and diverse transmission powers. So our study is focussed on Interweave approach in which secondary user only attempts to access the licensed bands when they are unoccupied by the Primary User.

In this paper cooperative spectrum sensing technique for cognitive radio is proposed based on M-ary QAM. 4-QAM, 8-QAM and 16-QAM is analysed in this study. Probability of False Alarms (Pfa) and Probability of Missed Detection (Pmd) and simulated to verify the results, These simulated results are plot to obtain the Receiver Operating Characteristics (ROC) curves. The rest of the paper is organised ass follows. In Section II, the Present-State-of-Research is discussed, focusing the merits and demerits of the present techniques and approaches within the defined area. Section III, describes the System Model and this mathematical Analysis, Section IV, the Simulation environment and simulation results. In Section V, Conclusion is drawn and in Section IV, Future Scope is entitled.

\section{Present State of Research}

Various work has been carried in this related field. Best research have been discussed here focusing the major achievement made and their gaps. Atapattu et al. in [2] analysed the performance of an cooperative energy detector for cognitive radio network to overcome the major problem associated with Non-Cooperative techniques i.e. multipath fading and shadowing. In another work Hussain et al. in [3] proposed Detect Amplify and Forward (DAF) relaying strategy for cooperative spectrum over non-identical Nakagami-m fading channels. In this few of the Cognitive Radio are incorporated with Cooperation and similar results is obtained compared to amplify-and-forward strategy in which all the Cognitive Radio are given Cooperative power. This technique reduces complexity and bandwidth utilization over the relaying links. Zheng et al. in [4] proposed a novel cooperative spectrum sensing algorithm to reduce network overheads. They also designed new mathematical expression for calculating Pfa and Pd and compared their results with the thresholds design method. Bagwari in [5] presented a technique in which the detection in performed in two stages for cognitive radio networks. For simple cases only stage one is used in which detection is based on single antenna with fixed threshold. For complex cases when the energy detected by the detector is close to the deciding threshold. In this case the second stage is required in which decision is made by adaptive double threshold technique. Abhijit Bhowmick et al. in [6] proposed in Improved Energy Detector (IED) for Cognitive Radios using Double Thresholds (DTHs). using this technique the no decision case when two $\underline{C R}$ node have different decision about the same channel. So to overcome this issue double threshold is used.

Ajay Singh et al. in [7] proposed cooperative spectrum sensing using multiple antennas. Using this technique reliability of spectrum sensing is improved with minimum level of 
interference. Wang et al. in [8] presented an optimal energy sensing algorithm based on Neyman-Pearson duality theorem for cooperative sensing using channel covariance matrix. Zhao et al. in [9] proposed energy efficient, quick and minimum overhead based Cooperative spectrum sensing technique. This involves two stages. To be quick and energy efficient only stage one is used, when Signal to Noise Ratio (SNR) is more or no primary user is present. However to increase accuracy stage two in required. For Cooperative the sharing of sensed data is sent using 1-bit decision this reduces overheads. $\mathrm{Vu}-\mathrm{Van}$ et al. in [10] proposes a cooperative spectrum sensing scheme in which low sensing data is sensed and based on the sensed data hybrid quantization is performed which minimizes the overheads and communication traffic. This novel approach enhances the overall performance of the Cognitive Radio system by reduces overheads and communication traffic. Huang et al. in [11] proposed wide-band spectrum analysis for cognitive radio. In wide-band to sense the whole spectrum band perfectly, Analog to Digital Converter (ADC) are required. To analysis such wide spectrum band complexity of the detector is increased exponentially. To overcome this a heuristic cooperative sensing algorithm is proposed, which reduces the computational complexity of the overall Cognitive Radio system. Lu et al. in [12] presented novel detecting model for the full duplex Transmitting Secondary Users, which have the capability to sense and transmit simultaneously. For the detection of spectrum holes an adaptive sensing window is also proposed, which improves accuracy of detection without any significant increase in system complexity. Afanan et al. in [15] enhanced the efficiency of spectrum utilization by proposing joint distributed beam-forming and cooperative relaying in Cognitive Radio Networks. In this approach beam-forming weights are calculated based on linear optimization to produce beam-forming by the reliable relay cognitive nodes. Chaves in [16] proposed an analytical expressions are developed for probability of detection for low sensing samples or for very low Signal to Noise Ratio levels.

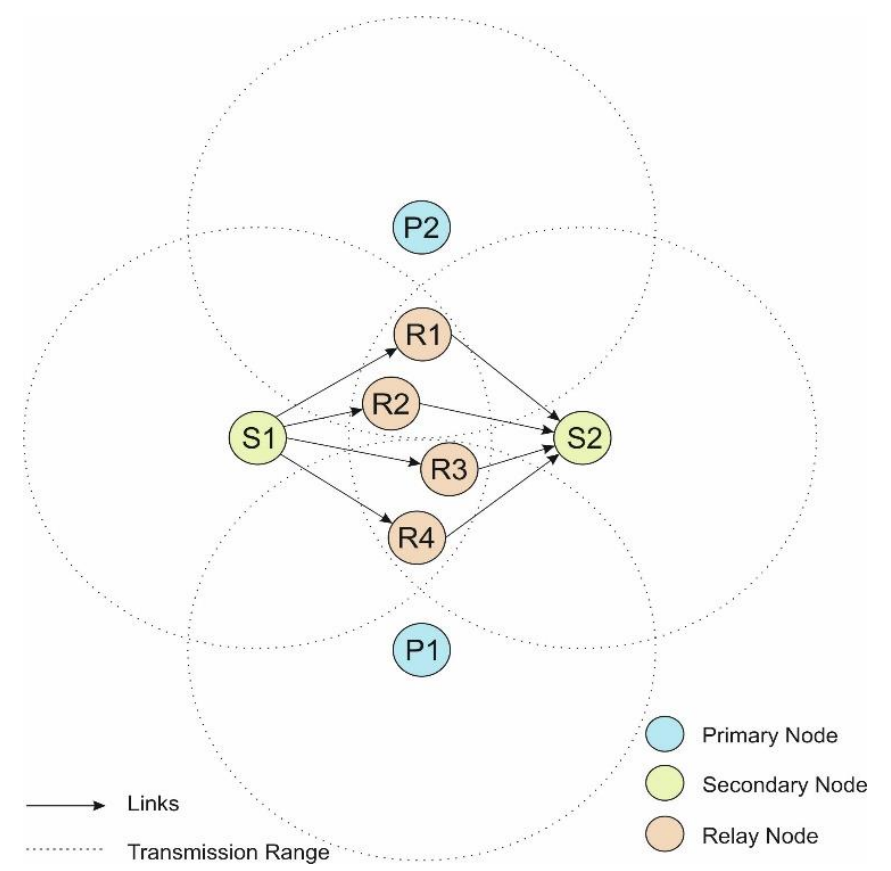

Figure 1. System Model 


\section{System Model}

To analyse the study Cognitive Radio network consisting of two Primary Users i.e PI and $P 2$, two Secondary or Cognitive Users $S 1$ and $S 2$ and four relay nodes i.e. R1 to R4. All these node are equipped with single antennas. All the four relay node fall within the transmission range of both the Secondary Users. Whereas the $R I$ and $R 2$ within the range of $2^{\text {nd }}$ Primary Users $P 1$ and $R 3$ an $R 4$ within the range of 1 st Primary User. Both the SU's are away from the transmission range of each other, therefore, no direct link can be set up between them. So the communication between them takes place using the relay nodes as shown in Figure 1.

In cooperative sensing technique, the sharing of sensed data is done by the detectors to overcome the problem of hidden node and shadowing. Moreover cooperation increase the efficiency of the system at the expense of bit complexity. So for precise system cooperation is used. In the proposed model each secondary user is equipped with energy detector and each SU detects the vacant bands. Sharing of the sensed data by each SU is forward to common centre called Fusion Centre where the final decision is made as shown in Figure 2. In this case $\$ m \$ S U$ are present and each user is equipped with energy detector. Every SU detects the vacant bands and forward the sensed data over to the Fusion Centre. The sensed data is in the binary for i.e 0 or $1 .{ }^{\prime} 0$ ' represent vacant band and ' 1 ' represents corresponding spectrum band is not vacant.

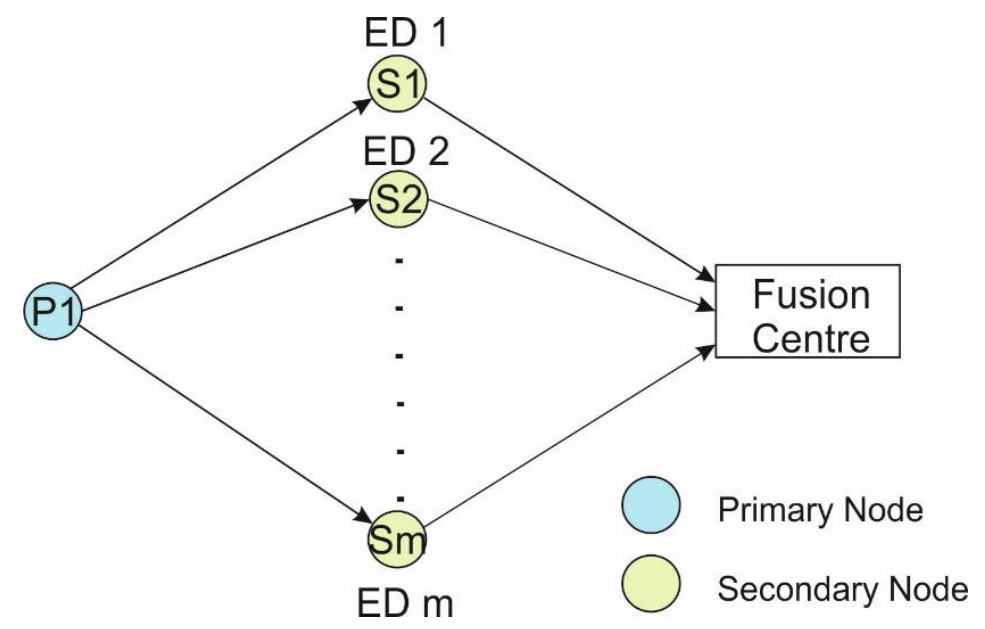

Figure 2. Cooperative Decision Making

\subsection{Energy Detection and Hypothetical Analysis}

The Energy Detector (ED) is a generally non-coherent detection technique in which prior knowledge of signal is not required. Therefore ED reduces he system cost and complexity but on the other hand produces the problem of Noise Uncertainty. This noise uncertainty cause issues like False Detections and Missed Alarms. For simulative testing the Energy Threshold level is calculated and the sensed energy by the ED is compared with this threshold. If sensed energy is less than the threshold the corresponding channel is idle (i.e. Primary User is Absent) and if sensed energy is greater than threshold energy the corresponding channel is busy (i.e. Primary User is Present)

$$
\begin{aligned}
& \text { Es }<\text { Eth Primary User is Absent } \\
& \text { Es }>\text { Eth Primary User is Present }
\end{aligned}
$$

The main objective to make decision whether Primary User is present or absent as shown in Equation. 1 and 2. In term of signal and noise the above equations can be also written as: -

$$
y(n)=u(n) \quad H_{0}: P U \text { is Absent }
$$




$$
y(n)=h(n) s(n)+u(n) \quad H_{1}: P U \text { is Present }
$$

Where 's(n)', is the transmitted signal, $\$ y(n)$ '\$ is the received signal over the fading channel ' $\mathrm{h}(\mathrm{n})$ ' having noise ' $\mathrm{u}(\mathrm{n})$ '. With the two outcomes i.e. Primary User Present or Absent, there are two error possibilities associated with it i.e. Probability of Missed Detection (Pmd) and Probability of False Alarm (Pfa).

For hypothetical testing of the system model, two hypothesis are considered $\mathrm{H}_{0}$ and $\mathrm{H}_{1}$. Hypothesis ' $\mathrm{H}_{0}{ }^{\prime}$ is when Primary User is not present i.e. the sensed energy is less than threshold and Hypothesis ' $\mathrm{H}_{1}$ ', when Primary User is Present i.e. sensed energy is greater than threshold. Under the Hypothesis ' $\mathrm{H}_{1}{ }^{\prime}$ if the energy is above calculated threshold Probability of Detection $(P d)$ is achieved and below threshold level Probability of Missed Detection Pmd is achieved, shown graphically in Figure 3a. Under the hypothesis ' $\mathrm{H}_{0}{ }^{\prime}$ if the energy is above threshold, wrong decision is made which is referred as Probability of False Alarm ( $P f a$ ) as shown in Figure 3b. This may be the case of high noise within the channel which deteriorate the SNR extremely.

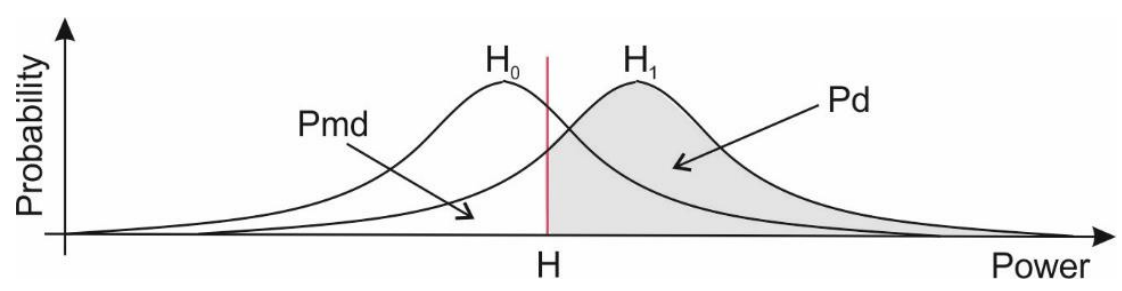

Figure 3a. Hypothetical Testing of Pmd and Pd

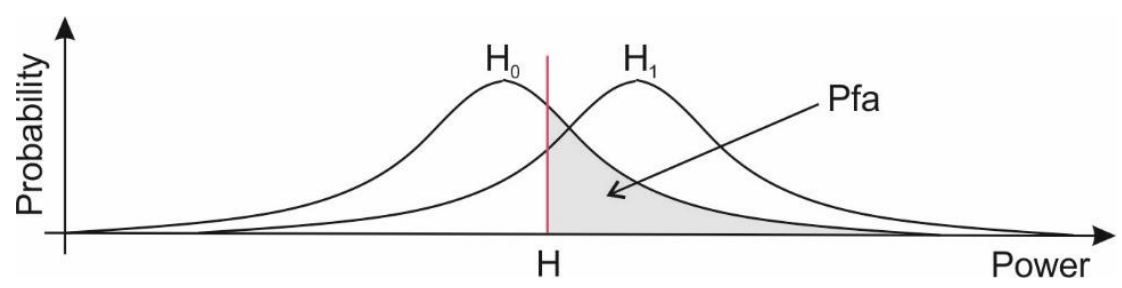

Figure 3b. Hypothetical Testing of Pfa

\subsection{Numerical Analysis}

Additive White Gaussian Noise (AWGN) where $\mathrm{x}(\mathrm{n})$ and $\mathrm{u}(\mathrm{n})$ represents their digital formats respectively, degrading the signal $x(t)$ having zero mean and variance $\sigma 2 \mathrm{n}$. According to the statistical properties of the output $y(t)$ or $y(n)$ threshold is set. The test statistic $y(t)$ is compared with the predefined threshold ' $\lambda$ ', which is calculated according to the formulation designed by Digham [16] using matlab gamma inverse cumulative distribution 'gaminv' function (cdf).

$$
\lambda(i)=\operatorname{gaminv}(1-P f a(i), m, 1) \times 2
$$

This function computes the inverse of gamma cdf having ' $\mathrm{N}$ ' as the sampling points and ' $\mathrm{m}=\frac{1}{2} \mathrm{~N}$ ' as the slope parameters and scale parameter fixed to unity, for corresponding probability set to 1 - Pfa having values between interval [0 1]. The gaminv function in terms of gamma cdf is mathematically linked as: -

$$
x=F^{-1}(p \mid a . b)=\{x: F(x \mid a, b)=p\}
$$

Where, $p$ is mathematically expressed as: -

$$
p=F(x \mid a, b)=\frac{1}{b^{a} \Gamma(a)} \int_{0}^{\infty} t^{a-1} e^{\frac{-x}{b}} d t
$$

and gamma cdf is formulated as: -

$$
[X, X L O, X U P]=\operatorname{gammacdf}(P, A, B, p, \text { cov, alpha })
$$

Where, ' $\mathrm{X}$ ' is defined as an output variable; whereas 'XLO' and 'XUP' contains its confidence lower and upper bounds respectively. Function on the whole produces the 
confidence bounds for ' $\mathrm{P}$ ' taking 'A' and 'B' as input estimates and 'p cov' containing input's covariance matrix of order $2 \times 2$ and 'alpha' fixed to 0.05 specifying, 100 (1 alpha)\% confidence bounds. Energy of the digitized received signal obtained using mathematical expression: -

$$
E(n)=\frac{\operatorname{sum}\left[a b s[y(n)]^{2}\right]}{\left.\operatorname{std}[n(t))^{2}\right]}
$$

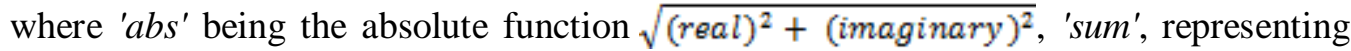
the sum of all the value sensed by the individual node stored in the form of row in the matrix and 'std' denoting the standard deviation, here being the standard deviation on AWGN of the channel. Comparison of this calculated energy ' $E(n)$ ' with the threshold ' $\lambda$ ', calculated earlier, ' $P d^{\prime}$ ' and ' $P f a$ ' is measured as:-

$$
P d=\frac{\frac{Q_{N}}{2}\left(\sqrt{\frac{\sqrt{(S N R}}{\sigma^{2}}} \cdot \sqrt{\frac{a}{\sigma^{2}}}, m\right)}{\Gamma\left(\frac{N}{2}\right)}
$$

where $\frac{Q_{N}}{2}$ is generalized Marcum-Q function of non-negative real numbers,$\sqrt{\frac{\propto(\text { SNR })}{\sigma^{2}}}$, $\sqrt{\frac{\lambda}{\sigma^{2}}}$ and $m$ being the positive integer defined in general as:

$$
Q=\operatorname{marcum} q(a, b, m)=\frac{1}{a^{m-1}} \int_{b}^{\infty} x^{m} \exp \left(-\frac{x^{2}+a^{2}}{2}\right) I_{m-1}(a x) d x
$$

where $I_{m-1}$ expresses modified Bessel function of first kind of order m-1.

$$
\text { Pf } a=\frac{\Gamma\left(\frac{N}{2}, \frac{\lambda}{2 \sigma^{2}}\right)}{\Gamma\left(\frac{N}{2}\right)}
$$

where $\Gamma$ represents incomplete gamma function, $\sigma$ standard deviation and $\sigma^{2}$ as variance. 'Pmd' can be calculated as:

$$
P m d=1-P d
$$

\section{Simulation Results}

MATLAB is used for the testing the proposed model. Receiver Operating Characteristics (ROC) curves show the relation between $P m d$ and $P f a$, is used for energy detection for detecting primary users in CR environment with $P f a$ on $\mathrm{X}$-axis and $P m d$ on Y-axis. While Energy Detectors have been extensively studied in the past [13-14] using BPSK and QAM signal, here ED using QAM is done which increases the system efficiency. Most of the proposed methods are ad hoc solutions and a more general model incorporating different parameters such as number of secondary users, detection and falsealarm probabilities and more importantly propagation characteristics is still lacking. Three different set-up has been taken to test and verify the proposed system. M-ary QAM modulation technique is used over different fading channels. Rician, Rayleigh and Addictive White Gaussian Noise (AWGN) fading channel are analysed in this research. Three different scenarios have been taken to verify the proposed model. 
SNR vs. Prob. of Missed Detection

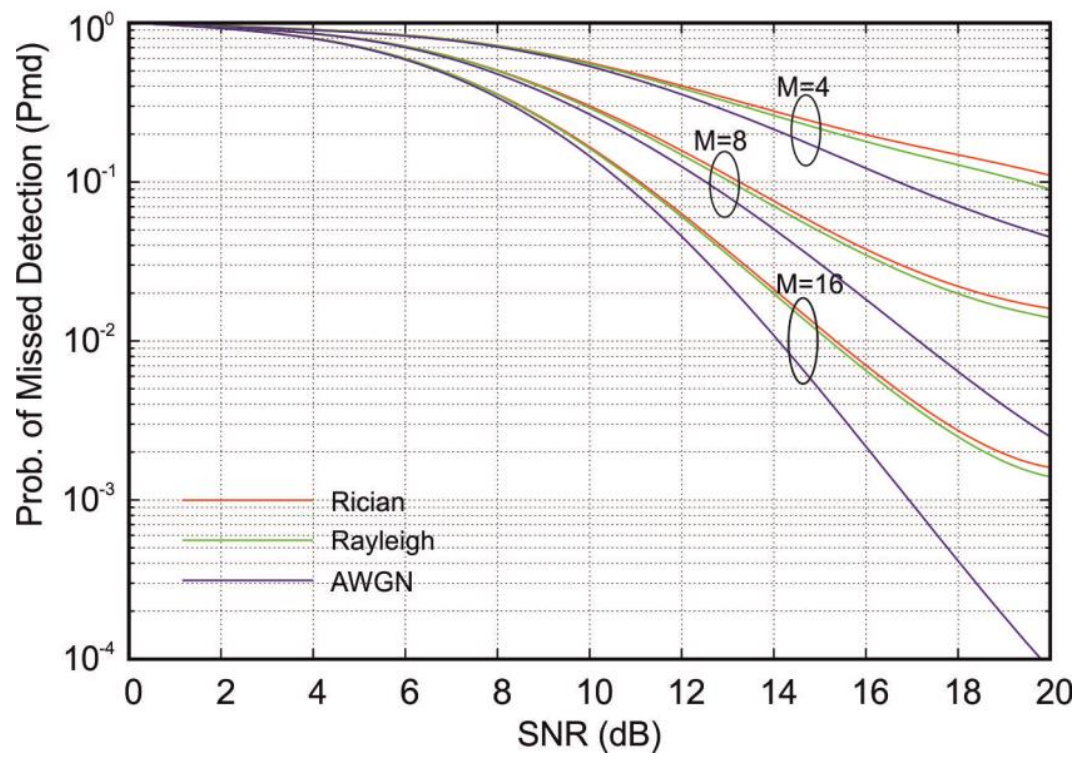

Figure 4. Pmd for Varying SNR over Different Fading Channels

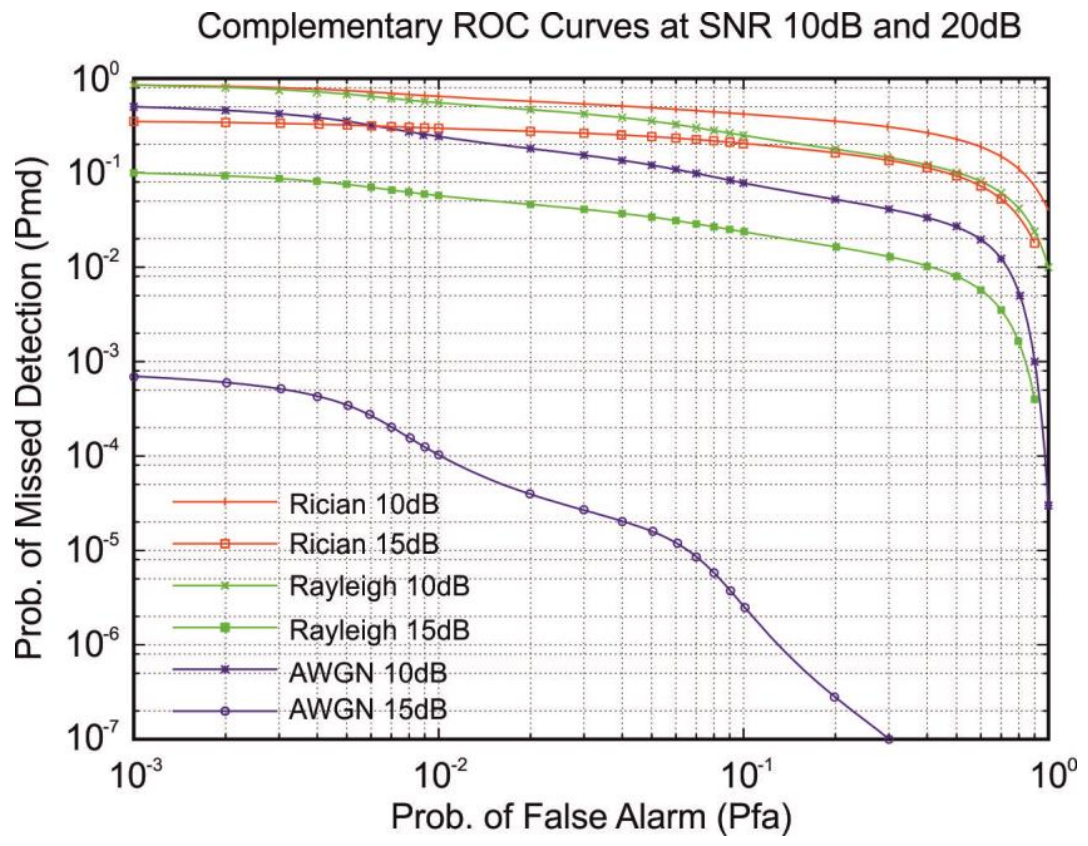

Figure 5. Pmd at $10 d B$ and $15 d B$ SNR

In the First case, Probability of Missed Detection (Pmd) for varying Signal to Noise Ratio $(S N R)$ is simulated for 4-QAM, 8-QAM and 16-QAM techniques. The simulation results for the same is plot as shown in Figure 4. From the graph it is clear that with the increase in SNR, Pmd decreases over all fading channels. Rician channel is considered as the worst channel as compared to the other channels. $M$ represents the level of QAM modulation used. With the increase in modulation level Pmd have further degraded, which shows enhancement of the system, as with the decrease in Pmd, Probability of Detection i.e. $P d$ increases. 32-QAM and 64-QAM was also simulated giving better results, but increases Latency period to more than $3 \mathrm{~ms}$, thereby making the system impractical to be used in real time scenario. So, the results for 32-QAM and 64-QAM are not included in this paper. 


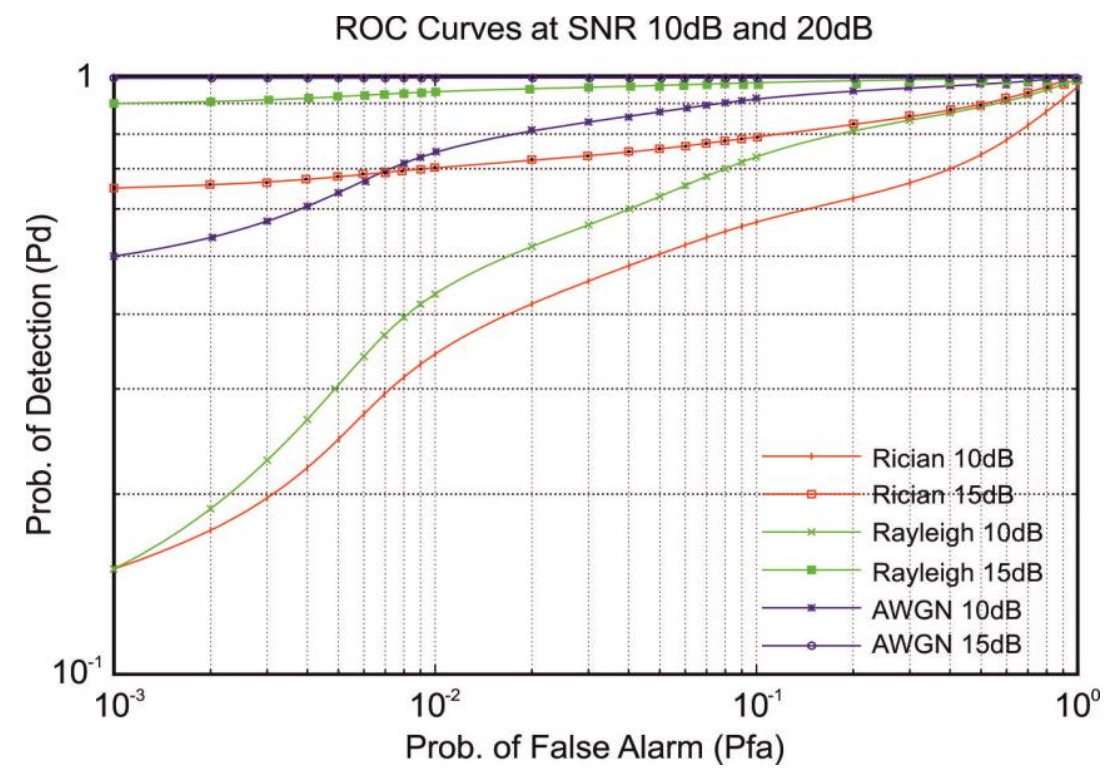

Figure 6. Pd at $10 d B$ and $15 d B$ SNR

In the Second case, Complimentary Receiver Operating Characteristic (ROC) curves have been simulated. In this $P f a$ vs $P m d$ is plotted for $10 \mathrm{~dB}$ and $15 \mathrm{~dB}$ SNR considering all the three fading channels as shown in Figure 5. In this case also Rician channel simulate out as the worst channel and AWGN with $15 d B$ as the best channel with least Pmd ranging from $9 \times 10^{-4}$ to $1 \times 10^{-7}$.

In the last case, ROC curves have been simulated, analysing the $P d$ with respect to $P f a$. In this $P d$ is calculated against $P f a$ for $10 d B$ and $15 d B$ SNR over all the three fading channels. The result for the same is plotted as shown in Figure 6. From the graph almost perfect sensing is achieved for AWGN channel at $15 d B$ SNR, with average $P d$ values at almost 1 , showing prefect sensing case. For AWGN channel with $10 d B$ SNR, minor degradation in the detection is noticed, representing degradation in detection due to noise.

\section{Conclusion}

In this paper M-ary QAM technique is used for Spectrum Sensing in Cognitive Radio Networks. Previous research shows that QAM performs better as compared to other modulation techniques for Cognitive Radio Spectrum Sensing. In this research sensing model based on M-ary QAM is proposed. 4, 8, 16 QAM is used to analyse and verify the Proposed Model. Simulation results shows that prefect sensing can be achieved using 16QAM where the environment conditions have SNR more than or equal to $15 d B$. However using 32 and 64 QAM even perfect detection of channel can be achieved at $10 \mathrm{~dB}$ and $5 \mathrm{~dB}$ SNR respectively at the expense of increase detection time. Results for the same are not incorporated as the sensing time for 32 and 64 QAM increases $\$ 3 \mathrm{~ms} \$$, thereby making the system impractical to be used in real time. 


\section{References}

[1] J. Mitola and G. Q. Maguire, “Cognitive Radio: Making Software Radio More Personal”, IEEE Personal Communications, vol. 6, no. 4, (1999), pp. 13-18, DOI: 10.1109/98.788210

[2] S. Atapattu, C. Tellambura and H. Jiang, "Energy Detection Based Cooperative Spectrum Sensing in Cognitive Radio Networks", IEEE Transactions on Wireless Communications, vol. 10, no. 4, (2011), pp. 1232 - 1241, DOI: 10.1109/TWC.2011.012411.100611.

[3] S. Hussain and X. N. Fernando, "Performance Analysis of Relay-Based Cooperative Spectrum Sensing in Cognitive Radio Networks Over Non-Identical Nakagami-m Channels", IEEE Transactions on Communications, vol. 62, no. 8, (2014), pp. 2733-2746, 2014. DOI: 10.1109/TCOMM.2014.2338856.

[4] X. Zheng, J. Wang, Q. Wu and L. Shen, "A Novel Cooperative Spectrum Sensing Algorithm in Cognitive Radio Systems", Journal of Communications and Networks, vol. 11, no. 2, (2009), pp. 115121, 2009. DOI: 10.1109/JCN.2009.6391386.

[5] A. Bagwari, G. S. Tomar and S. Verma, "Cooperative Spectrum Sensing Based on Two-Stage Detectors With Multiple Energy Detectors and Adaptive Double Threshold in Cognitive Radio Networks", Canadian Journal of Electrical and Computer Engineering, IEEE, vol. 36, no. 4, (2014), pp. 172-180, 2013. DOI: 10.1109/CJECE.2014.2303519.

[6] A. Bhowmick, A. Chandra, S. D. Roy and S. Kundu, "Double Threshold Based Cooperative Spectrum Sensing for Cognitive Radio Network with Improved Energy Detectors", IET Communications, vol. 9, no. 18, (2014), pp. 2216-2226, 2015. DOI: 10.1049/iet-com.2014.1098.

[7] A. Singh, M. R. Bhatnagar and R. K. Mallik, "Cooperative Spectrum Sensing in Multiple Antenna Based Cognitive Radio Network Using an Improved Energy Detector", IEEE Communications Letters, vol. 16, no. 1, (2012), pp. 64-67, DOI: 10.1109/LCOMM.2011.103111.111884.

[8] H. Wang, X. Su, Y. Xu, S. Zhou and J. Wang, "Optimal cooperative energy spectrum sensing in cognitive radio network", Frontiers of Electrical and Electronic Engineering in China, vol. 5, no. 4, (2010), pp. 449-455, DOI: 10.1007/s11460-010-0113-z.

[9] N. Zhao, F. R. Yu, H. Sun and A. Nallanathan, "Energy Efficient Cooperative Spectrum Sensing Schemes for Cognitive Radio Networks", EURASIP Journal on Wireless Communications and Networking, (2013), DOI: 10.1186/1687-1499-2013-120.

[10] H. Vu-Van and I. Koo, "Cooperative Spectrum Sensing Using Individual Sensing Credibility and Hybrid Quantization for Cognitive Radio", Intelligent Information and Database Systems, vol. 6591 of the series Lecture Notes in Computer Science, (2011), pp. 528-537. DOI: 10.1007/978-3-642-20039$7 \backslash 53$.

[11] H. Huang, Z. Y. Zhang, P. Cheng, A. P. Huang and P. L. Qiu, "Cooperative Spectrum Sensing in Cognitive Radio Systems with Limited Sensing Ability", Journal of Zhejiang University SCIENCE C, vol. 11, no. 3, (2010), pp. 175-186, DOI: 10.1631/jzus.C0910027.

[12] Y. Lu, D. Wang, M. Fattouche, "Cooperative Spectrum Sensing Algorithm in Cognitive Radio by Simultaneous Sensing and BER Measurements", EURASIP Journal on Wireless Communications and Networking, (2016), DOI: 10.1186/s13638-016-0635-z.

[13] J.S.P. Singh, R. Singh, M.K. Rai, J. Singh and A.S. Kang, "Cooperative Sensing for Cognitive Radio: A Powerful Access Method for Shadowing Environment", Wireless Personal Communication, vol. 80, no. 4, (2014), pp. 1363-1379, DOI: 10.1007/s11277-014-2088-1.

[14] J.S.P. Singh and M.K. Rai, "Cognitive Radio Intelligent-MAC (CR-i-MAC): Channel-Diverse Contention Free Approach for Spectrum Management", Telecommunication Systems, (2016), DOI: 10.1007/s11235-016-0188-9.

[15] A. Afana, V. Asghari, A. Ghrayeb and S. Affes, "On the Performance of Cooperative Relaying Spectrum Sharing Systems with Collaborative Distributed Beam-forming", IEEE Transaction on Communications, vol. 62, no. 3, (2014), pp. 857-871, DOI: 10.1109/TCOMM.2014.032014.130399

[16] D. S. A. Chaves and A. N. Barreto, "Energy Detection Spectrum Sensing of M-ary QAM systems over AWGN Channels", International Symposium on Wireless Communication Systems, IEEE, (2012), pp. 106-110. DOI: 10.1109/ISWCS.2012.6328339

\section{Authors}

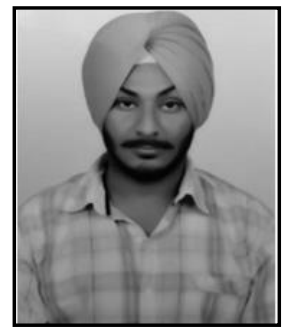

Jai Sukh Paul Singh, working as a Research Scholar (Ph.D.) from Lovely Professional University, Punjab India and did his B.Tech from Chandigarh Engineering College, Landran (2011) in Electronics and Communication Engineering, M.Tech from Guru Nanak Dev University, Amritsar in 2013 with area of specialization in Cognitive Radio Technology, Spectrum sensing, Communication Systems, Wireless Comm. Wireless Sensor Networks and Signal Processing. $\mathrm{He}$ is the Member of IEEE-India, Delhi Section, Member ComSoc. 


\begin{abstract}
He has many publication in well-renowned International journals and Conferences.
\end{abstract}

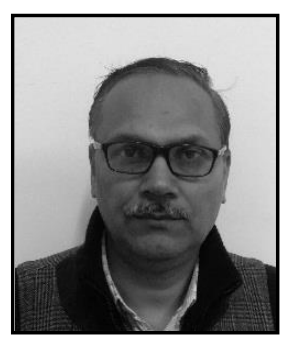

Mritunja Kumar Rai, received his Doctorate degree from ABVIndian Institute of Information Technology and Management, Gwalior, India, after the completion the Master of Engineering degree in Digital system form Motilal Nehru National Institute of Technology, Allahabad, India. Presently he is working as an Associate Professor in Lovely Professional University, Phagwara, India. His research Interest include wireless networks and Network Security. He has published more than 30 research articles in reputed International Conferences and International Journals.

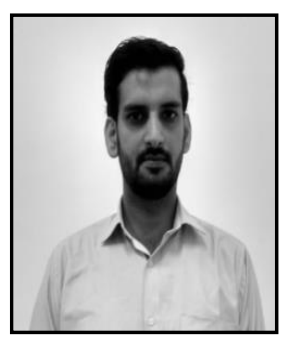

Gulshan Kumar, working as an Assistant Professor in Lovely Professional University, Punjab India and did his B.Tech from Amritsar College of Engineering, Amritsar (2009) in Computer Science Engineering, M.Tech from Lovely Professional University, Punjab, India with area of specialization in Mobile Ad hoc and Sensor Networks. He has many publication in well renowned International journals and Conferences.

Hye-jin Kim, is presently working in Business Administration Research Institute, Sungshin W. University, Seoul, Republic of Korea. She has published a number of research papers in various International Journals. 